\title{
Training of health agents for a better approach hypertensive patient
}

\section{Capacitação das agentes de saúde para melhor Abordagem ao paciente hipertenso}

DOI: $10.46919 / \operatorname{archv1n6-009}$

Recebimento dos originais: 01/09/2020

Aceitação para publicação: 30/10/2020

\section{Arantxa Palhares Marinho}

Graduada em Medicina pelo IMEPAC

Instituição: Instituto Master de Ensino Presidente Antonio Carlos

Endereço: Avenida Minas Gerais 1889- Centro Araguari, MG CEP 38440-148

E-mail: arantxa_palhares@hotmail.com

\section{Camila Caetano de Paula Miranda Valladares}

Graduada em Medicina pelo IMEPAC

Instituição: Instituto Master de Ensino Presidente Antonio Carlos

Endereço: Avenida Minas Gerais 1889- Centro Araguari, MG CEP 38440-148

E-mail:mila_miranda_@hotmail.com

Daniel Almeida Silqueira

Graduado em Medicina pelo IMEPAC

Instituição: Instituto Master de Ensino Presidente Antonio Carlos

Endereço: Avenida Minas Gerais 1889- Centro Araguari, MG CEP 38440-148

E-mail: daniel.silqueira@hotmail.com

\section{Daniel André}

Graduado em Medicina pelo IMEPAC

Instituição: Instituto Master de Ensino Presidente Antonio Carlos

Endereço: Avenida Minas Gerais 1889- Centro Araguari, MG CEP 38440-148

E-mail: daniel.silqueira@ hotmail.com

\section{Gabriela Vilaça Romero Duarte}

Graduada em Medicina pelo IMEPAC

Instituição: Instituto Master de Ensino Presidente Antonio Carlos

Endereço: Avenida Minas Gerais 1889- Centro Araguari, MG CEP 38440-148

E-mail: gabivilaca@msn.com

\section{Gustavo Prado Pouzas Guedes}

Graduado em Medicina pelo IMEPAC

Instituição: Instituto Master de Ensino Presidente Antonio Carlos

Endereço: Avenida Minas Gerais 1889- Centro Araguari, MG CEP 38440-148

E-mail: gpradopouzas@gmail.com 


\title{
Julio Cezar Viana Dias
}

Graduado em Medicina pelo IMEPAC

Instituição: Instituto Master de Ensino Presidente Antonio Carlos

Endereço: Avenida Minas Gerais 1889- Centro Araguari, MG CEP 38440-148

E-mail: juliocezarvianadias@hotmail.com

\section{Marcelo Augusto Agne Neuwald}

Graduado em Medicina pelo IMEPAC

Instituição: Instituto Master de Ensino Presidente Antonio Carlos

Endereço: Avenida Minas Gerais 1889- Centro Araguari, MG CEP 38440-148

E-mail:marceloneuwald@hotmail.com

Mayanderson Rosa da Silva

Graduado em Medicina pelo IMEPAC

Instituição: Instituto Master de Ensino Presidente Antonio Carlos

Endereço: Avenida Minas Gerais 1889- Centro Araguari, MG CEP 38440-148

E-mail:mayanderson18@hotmail.com

\section{Letícia Rosa Santos}

Graduada em Enfermagem e Obstetricia pela Fundação Uniersidade Federal do Rio Grande -FURGInstituição: Universidade de Brasilia

Endereço: UnB - Brasília, DF, 70910-900

E-mail: tenenteleticia@yahoo.com.br

\section{RESUMO}

Diante da relevância da Hipertensão Arterial Sistêmica, considerada como um dos principais problemas de saúde pública e de suas temidas complicações, tornou-se viável planejar uma estratégia como medida de intervenção, orientando as agentes de saúde quanto as suas temidas complicações e agravantes, para melhor abordagem aos pacientes com doença hipertensiva daquela área.

Foi observado na Unidade de Saúde da Família do bairro Novo Horizonte do município de Araguari-MGque $25 \%$ da população idosa era hipertensa.

Estudos em geral, indicam que os índices de pacientes hipertensos controlados são baixos e estão relacionados principalmente a aderência ao tratamento - tanto farmacológico quanto não farmacológicoSendo assim, foi ministrado um curso de capacitação para melhor abordagem ao paciente hipertenso, onde foram abordados os seguintes tópicos: conceito de hipertensão, sinais e sintomas, consequências e complicações caso o paciente não realize a adesão ao tratamento, medicamentos utilizados, valores de pressão considerados normais para cada faixa etária, qual a melhor atitude a ser tomada na ausência de adesão ao tratamento, como evitar o desenvolvimento da doença hipertensiva e fatores pré-disponíveis para hipertensão abordando hábitos de vida assim como fatores genéticos.

O intuito do curso ofertado foi de sensibilizar e orientar as agentes de saúde para melhor abordagem aos pacientes hipertensos daquela unidade.

Palavras-Chave: Capacitação, idosos, hipertensão, agentes de saúde.

\begin{abstract}
In view of the relevance of Systemic Arterial Hypertension, considered as one of the main public health problems and its dreaded complications, it became feasible to plan a strategy as an intervention measure,
\end{abstract}


guiding health agents regarding their feared complications and aggravations, for better approach to patients with hypertensive disease in that area.

It was observed at the Family Health Unit in the Novo Horizonte neighborhood of the city of AraguariMG- that $25 \%$ of the elderly population was hypertensive.

Studies in general indicate that the rates of controlled hypertensive patients are low and are mainly related to treatment adherence - both pharmacological and non-pharmacological-

Therefore, a training course was given to better approach the hypertensive patient, where the following topics were addressed: concept of hypertension, signs and symptoms, consequences and complications if the patient does not adhere to treatment, medications used, pressure values considered normal for each age group, what is the best attitude to be taken in the absence of adherence to treatment, how to avoid the development of hypertensive disease and pre-available factors for hypertension addressing lifestyle habits as well as genetic factors.

The purpose of the offered course was to raise awareness and guide health agents to better approach hypertensive patients in that unit.

Key Words:Training, elderly, hypertension, health agents

\section{INTRODUÇÃO}

A hipertensão arterial vem se transformando progressivamente num dos mais graves problemas de saúde pública, atingindo adultos, em especial os mais idosos, sendo definida pela Organização Mundial de Saúde como a elevação crônica da pressão arterial sistólica e/ou pressão arterial diastólica. Sua prevalência tende a ser maior no sexo masculino. Os números são muito variáveis para o país, variando de $5,0 \%$ a $32,7 \%$ em diferentes regiões do país. A não adesão do tratamento medicamentoso da hipertensão arterial pelos idosos é um dos principais problemas encontrados pelos profissionais que trabalham na atenção primária. Para que a assistência prestada a esses pacientes seja eficaz é necessário que os agentes estejam preparados para lidar com esta problemática.

\section{OBJETIVO}

Capacitar as agentes de saúde do Posto do bairro Novo Horizonte para melhor atender os idosos hipertensos moradores da comunidade.

\section{MÉTODOS}

A pesquisa-ação é um tipo de pesquisa social com base empírica que é concebida e realizada em estreita associação com uma ação ou com a resolução de um problema coletivo. Para primeira etapa da pesquisa-ação selecionou-se o bairro Novo Horizonte localizado no município de Araguari, pois aproximadamente $25 \%$ da população assistida pela Estratégia de Saúde da Família do bairro apresenta hipertensão arterial sistêmica. Segundo relato dos profissionais atuantes nessa estratégia, não existe uma correta adesão ao tratamento por parte desses pacientes .Perante essa realidade, o mais adequado é 
ministrar cursos de capacitação para os agentes de saúde locais uma vez que, é de grande importância que os pacientes compreendam o quanto é fundamental a adesão ao tratamento. No curso de capacitação a ser ministrado abordaremos os seguintes temas: conceito de hipertensão, sinais e sintomas, conseqüências e complicações caso o paciente não trate, medicamentos utilizados, valores pressóricos considerados normais para cada faixa etária e sexo, qual a melhor atitude a ser tomada na ausência da adesão ao tratamento, como evitar a hipertensão, fatores pré-disponentes a hipertensão abordando hábitos de vida e fatores genéticos.

\section{CONCLUSÃO}

Tal trabalho originou-se a partir da demanda das ACS- Agentes Comunitárias de Saúde frente ao grande número de idosos hipertensos cadastrados na estratégia de saúde da família do bairro Novo Horizonte, e na sequência a percepção da falta de cuidado que estes têm com a adesão ao tratamento farmacológico e não farmacológico.

Observamos ainda que, por mais que o tema Hipertensão Arterial possa parecer fácil de ser trabalhado, muitas das agentes de saúde possuíam dificuldade em abordar o tema com os usuáriosprincipalmente os não aderentes ao tratamento- elaborando dessa forma estratégias de ação/prevenção e educação em saúde.

As informações que um usuário recebe podem contribuir na compreensão do processo de adoecimento, causas e consequências de sua comorbidade crônica. Ajudando na prevenção de doenças e em mudança de estilo de vida com intuito de manter o organismo saudável e o mais blindado possível contra suas complicações.

Capacitar as agentes de saúde, nos levou a refletir melhor sobre o tema, e perceber o quão importante é deixar aqueles profissionais que estão em um contato maior com a população adscrita bem orientados quanto aos complicadores oriundos de uma Hipertensão Arterial Sistêmica mal controlada. Assim, ampliamos nossa roda de transmissores do saber e aumentamos as chances de os pacientes passarem a aderir ao tratamento proposto com mais responsabilidade. Dessa forma, pretendemos diminuir a parcela de usuários que buscam unidades de urgência/emergência devido a complicações agudas da hipertensão arterial e, consequentemente, melhorar sua qualidade de vida.

Esperamos que este plano de ação, possa construir um conhecimento compartilhado para uma assistência mais adequada e uma comunidade com mais conhecimento, consciente e comprometida com seu processo de saúde/doença. Uma atenção contínua e eficaz para a saúde e o bem-estar da população requer diferentes níveis de intervenção dos serviços de saúde, adequados ás distintas fases da enfermidade e ao grau de incapacidades (BRASIL, 2007b) 


\section{REFERÊNCIAS}

DANTAS, André de Oliveira.Hipertensão Arterial no idoso: Fatores

dificultadores para adesão ao tratamento medicamentoso. [especialização].

Universidade Federal de Minas Gerais; 2011. 31 p.

MELLO, C. Métodos Qualitativos: pesquisa-ação; [internet]. Disponivel em: http://www.carlosmello.unifei.edu.br/Disciplinas/Mestrado/PCM-10/SlidesMestrado/Metodologia_Pesquisa_2012-Slide_Aula_11_Mestrado.pdf. Acessado em: $13 / 03 / 2013$

THIOLLENT, M. Metodologia da Pesquisa-ação. 14TM edição. São Paulo: Cortez Editora, 2005.

Brasil. Ministerio da Saúde. Secretaria de Atençao a Saude. Departamento da Atençao Basica. Envelhecimento da pessoa idosa. Brasilia: Ministerio da Saude,2007b. Disponivel em httpp://bvsms.saude.gov.br/bvs/publicações/abcad19.pdf. Acesso em 2013. 